Seyed Mohammad Safieddin Ardebili, Xinyu Ge and Giancarlo Cravotto*

\title{
Flow-mode biodiesel production from palm oil using a pressurized microwave reactor
}

https://doi.org/10.1515/gps-2017-0116

Received August 3, 2017; accepted December 14, 2017; previously published online February 12, 2018

\begin{abstract}
The factors that influence microwave-assisted biodiesel production reactions have been analyzed in this investigation. The studied parameters included microwave (MW) power, irradiation time, and reactor pressure. The response surface method was used to optimize the reaction conditions. The conversion for the $6: 1$ methanol/oil molar ratio and $1 \%$ catalyst ranged from $68.4 \%$ to $96.71 \%$. The optimized conditions were found to be $138 \mathrm{~s}$ of MW irradiation at $780 \mathrm{~W}$ and 7 bar pressure. The conversion at this point was $97.82 \%$. Biodiesel yield increased at higher radiation times (90-130 s) and pressures (5-7 bar). Results show that MW power and irradiation time have significant effects at the $1 \%$ level, whereas pressure had significant effects at the $5 \%$ level on biodiesel production in this range. The major properties of the palm oil biodiesel produced herein have met the requirements of the EN 14214 methyl ester standard.
\end{abstract}

Keywords: biodiesel; flow process; microwave-assisted transesterification; pressurized microwave reactor; response surface method.

\section{Introduction}

Energy is a basic need for humankind. Pollution from fossil fuels has led to global warming and climate change $[1,2]$. Biodiesel is a methyl/ethyl ester produced from vegetable oils or animal fat, which can be used as fuel in diesel engines or thermal systems [3]. Biodiesel can be extracted from waste vegetable oils as well as from straight vegetable oils $[4,5]$.

\footnotetext{
*Corresponding author: Giancarlo Cravotto, Dipartimento di Scienza e Tecnologia del Farmaco and NIS, Centre for Nanostructured Interfaces and Surfaces, University of Turin, via P. Giuria 9, 10125 Turin, Italy, e-mail: giancarlo.cravotto@unito.it

Seyed Mohammad Safieddin Ardebili: Department of Biosystems Engineering, Shahid Chamran University of Ahvaz, Ahvaz, Iran Xinyu Ge: Dipartimento di Scienza e Tecnologia del Farmaco and NIS, Centre for Nanostructured Interfaces and Surfaces, University of Turin, via P. Giuria 9, 10125 Turin, Italy
}

Ә Open Access. (c) BY (c) 2019 Walter de Gruyter GmbH, Berlin/Boston
We have compared classic biodiesel production by transesterification under conventional heating with processes in loop reactors, as assisted by ultrasound [6,7], hydrodynamic cavitation [8], and continuous-flow microwaves (MW) $[9,10]$. It was found that efficient heat and mass transfer and catalyst loading significantly influence the transesterification rate [11]. Hybrid reactors that combine acoustic cavitation and dielectric heating have therefore proven themselves to be highly efficient [12, 13].

MW heating significantly enhances transesterification, whereas volumetric heating provides several advantages, such as smaller reacting vessel, shorter reaction times, lower alcohol/oil ratios, and energy savings [13-15]. MW-assisted biodiesel production has been reported both under basic [16] and acid catalysis [17], but has also been carried out under heterogeneous catalysts [18] and in enzymatic processes [19]. Furthermore, fast conversions (94\%) have been obtained in $30 \mathrm{~s}$ of irradiation ( $800 \mathrm{~W}$ ) in a 9:1 molar ratio and oil/sodium hydroxide (1\%) [20]. Several other studies have demonstrated the advantages of dielectric heating in waste oil transesterification [21-24].

The economic aspects have been reported in another study; 3 min were required to produce 1:6 molar ratio biodiesel under $750 \mathrm{~W}$ MW exposure. In fact, the results showed that MW-assisted biodiesel production (as a fuel for power generators) is cost-effective [25]. The studied parameters included MW irradiation time, reaction time, and MW power. The response surface method (RSM) was used to optimize the reaction conditions [26-29].

\section{Materials and methods}

\subsection{Materials}

Palm oil, supplied by Oilitalia Co. Ltd. (Italy), was used as the raw material for biodiesel production. The GC-MS analyses of palm oils are shown in Figure 1. Methanol (99.9\%), potassium hydroxide flakes (85\%), and $n$-heptanes were obtained from Sigma-Aldrich (Milan, Italy) and used without further purification.

\subsection{Equipment}

The transesterification reaction was performed under MW irradiation using sodium methoxide as the catalyst. The catalyst solution was 


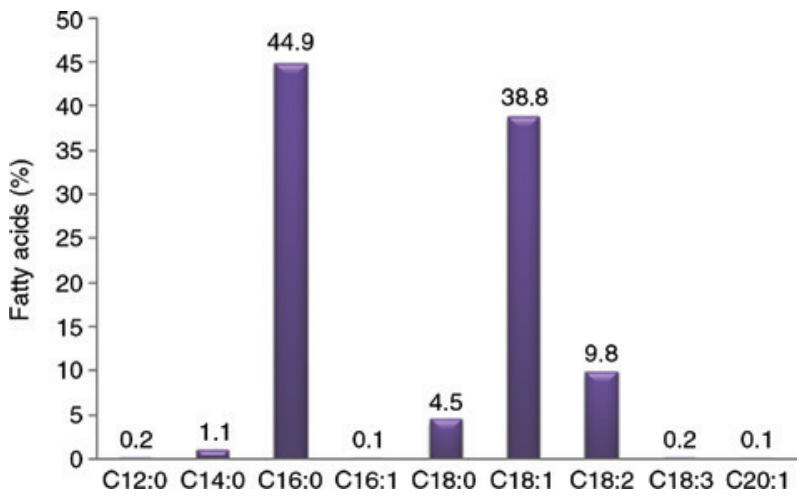

Figure 1: Palm oil fatty acids profile.

prepared using a magnetic stirrer. The experimental setup consisted of a continuous-flow MW reactor apparatus (Milestone FlowSynth). Figure 2 shows the setup that was used in this work.

This system incorporated a variable-power MW oven that could control temperature, MW power, and pressure inside the reactor. The maximum working temperature of the system was $200^{\circ} \mathrm{C}$. The reactor was equipped with a $250 \mathrm{ml}$ cylindrical polytetrafluoroethylene vessel with an internal rotating mixer. The reaction mixture was pumped through the reactor. The maximum working pressure was $30 \mathrm{bar}$ and the flow rate was $100 \mathrm{ml} / \mathrm{min}$. The effects of MW power (200-1000 W), MW irradiation time (60-180 s), and reaction pressure (5-15 bar) were evaluated in this work.

A 6:1 molar ratio and 1\% catalyst loading were selected. The tests were primarily carried out under software-recommended conditions. All samples were then exposed to $6000 \mathrm{rpm}$ of centrifugal motion for $5 \mathrm{~min}$ to separate the biodiesel phase. Methyl ester content (\%) in biodiesel was measured using Equation 1 . The free fatty acid content of samples was determined using methyl heptadecanoate and normal heptane solutions [22]:

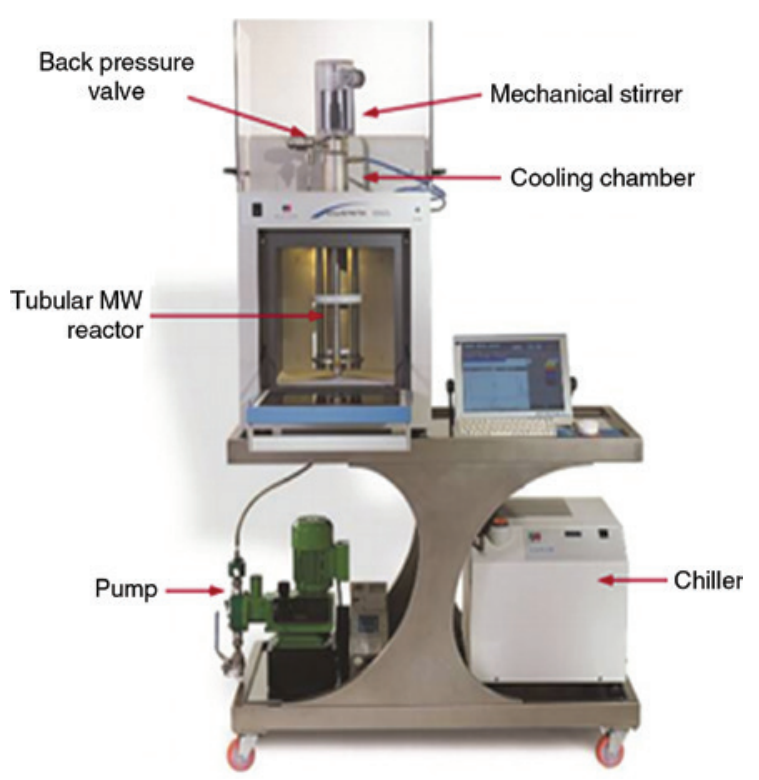

Figure 2: Continuous-flow MW reactor for biodiesel production.

$$
\% \text { of } \mathrm{ME}=\frac{\sum A-A_{E I}}{A_{E I}} \times \frac{C_{E I} \times V_{E I}}{\mathrm{~m}} \times 100
$$

$\Sigma A=$ total peak area from the methyl ester on $\mathrm{C}_{14}$ to the one on $\mathrm{C}_{24}$ $A_{E I}=$ methyl heptadecanoate peak area

$C_{E I}=$ methyl heptadecanoate solution concentration $(\mathrm{mg} / \mathrm{ml})$

$V_{E I}=$ methyl heptadecanoate solution volume (ml)

$\mathrm{m}=$ mass of the sample $(\mathrm{mg})$.

The glycerin phase was separated from the biodiesel, which was then leached. All experimental conditions were investigated three times and mean values were entered into the software.

\subsection{Experimental design}

RSM is an effective statistical method used to study complex processes with no fully known mechanism. The relationship between independent variables and responses was determined using the central composite design with three replications. The central composite design provides the advantage of requiring fewer experiments [30]. Design Expert 7.0.0 was used for RSM data analysis purposes. This method was adopted due to the ease with which the catalyst content (\%) could be selected. After carrying out the experiments and calculating reaction efficiency, the optimal points of the process were obtained by selecting the proper weights and variation ranges. The corresponding values were then evaluated by repeating the experiment at the recommended optimal point. After finding the optimum conditions, the effective parameters for the transesterification reaction were analyzed in this state. Biodiesel production efficiency was selected as the response parameter. Study variables and levels are presented in Table 1.

The operating pressure conditions are a continuation of those from Choedkiatsakul et al. [9], as the temperature maximum described in their article is related to the minimum pressure used in this investigation.

Once data were entered into the software, the second-order model was fitted to find the relationship between the response variable (biodiesel efficiency) and the independent variable. The general form of the second-order polynomial equation is expressed by Equation 2.

$$
Y=\beta_{0}+\sum_{i=1}^{k} \beta_{i} X_{i}+\sum_{i=1}^{k} \beta_{i i} X_{i}^{2}+\sum_{i=1}^{k} \sum_{j=i+1}^{k} \beta_{i j} X_{i} X_{j}+\varepsilon
$$

where $Y$ is the response (dependent variable), which is the biodiesel conversion percentage in this case, $\beta_{0}$ is a constant, $\beta_{i}, \beta_{i j}$, and $\beta_{i j}$ are the linear and square coefficients and the mutual effect of parameters, respectively, whereas $\varepsilon$ denotes the prediction error. $X_{i}$ and $X_{j}$ are the independent variables: $\mathrm{MW}$ irradiation time, pressure, and MW power in this case [31].

Table 1: Independent variables for central composite design (CCD).

\begin{tabular}{lrrrrll}
\hline \multicolumn{3}{l}{ Codes factor levels } & & & $\boldsymbol{X}_{\boldsymbol{i}}$ & Independent variable \\
\cline { 1 - 1 } $\boldsymbol{\alpha}$ & -1 & $\mathbf{0}$ & +1 & $+\boldsymbol{\alpha}$ & & \\
\hline 60 & 90 & 120 & 150 & 180 & $X_{1}$ & MW irradation time (S) \\
2 & 5 & 8 & 11 & 14 & $X_{2}$ & Pressure (bar) \\
200 & 400 & 600 & 800 & 1000 & $X_{2}$ & MW power (W) \\
\hline
\end{tabular}




\section{Results and discussion}

\subsection{Experimental design and statistical analysis}

The effect that a number of parameters (namely, MW irradiation time, power, and reaction pressure) have on the transesterification reaction and the MW-assisted production efficiency of methyl esters has been investigated in this study. Twenty experiments with three replications were required to optimize the chemical parameters of the reaction, using RSM for three parameters at five levels. Table 2 presents the empirical results of the experiments. The conversion for $6: 1$ molar ratio and $1 \%$ catalyst ranged from $68.4 \%$ to $96.71 \%$.

Analysis of variance (ANOVA) results show that the effects of MW irradiation time and power were significant at the $1 \%$ level of significance, as seen in Table 3. These significant effects indicate how important and effective the selected independent variables are for the experiments. ANOVA was selected to analyze the significant effects of the process variables on the response. As shown in the ANOVA table, the lack-of-fit test was nonsignificant for the study data. This indicates that the model predicted the data trends well [26].

The statistical significance of both effects (power and irradiation time) suggest that the selected

Table 2: Central composite design for three-factor coded conditions.

\begin{tabular}{lrrrrr}
\hline Run & Std & $\begin{array}{r}\text { Microwave } \\
\text { irradiation time (s) }\end{array}$ & $\begin{array}{r}\text { Pressure } \\
\text { (bar) }\end{array}$ & $\begin{array}{r}\text { Power } \\
\text { (W) }\end{array}$ & $\begin{array}{r}\text { Conversion } \\
\text { (\%) }\end{array}$ \\
\hline 1 & 13 & 0 & 0 & -2 & 68.4 \\
2 & 16 & 0 & 0 & 0 & 96.08 \\
3 & 17 & 0 & 0 & 0 & 92.84 \\
4 & 14 & 0 & 0 & 2 & 96.71 \\
5 & 8 & 1 & 1 & 1 & 96.02 \\
6 & 3 & -1 & 1 & -1 & 77.21 \\
7 & 1 & -1 & -1 & -1 & 75.06 \\
8 & 19 & 0 & 0 & 0 & 94.02 \\
9 & 6 & 1 & -1 & 1 & 94.05 \\
10 & 4 & 1 & 1 & -1 & 84.1 \\
11 & 20 & 0 & 0 & 0 & 92.03 \\
12 & 11 & 0 & -2 & 0 & 89.34 \\
13 & 7 & -1 & 1 & 1 & 92.81 \\
14 & 5 & -1 & -1 & 1 & 89.03 \\
15 & 15 & 0 & 0 & 0 & 93.92 \\
16 & 2 & 1 & -1 & -1 & 82.79 \\
17 & 12 & 0 & 2 & 0 & 96.18 \\
18 & 18 & 0 & 0 & 0 & 94.71 \\
19 & 9 & -2 & 0 & 0 & 85.2 \\
20 & 10 & 2 & 0 & 0 & 90.15 \\
\hline & & & & &
\end{tabular}

Table 3: ANOVA for coefficients of the RSM.

\begin{tabular}{lrrrrr}
\hline Source & $\boldsymbol{d f}$ & Sum of square & Mean square & F value & p-Value \\
\hline Model & 9 & 1134.19 & 126.02 & 21.67 & $<\mathbf{0 . 0 0 0 1}$ \\
$X_{1}$ & 1 & 67.04 & 67.04 & 11.53 & $\mathbf{0 . 0 0 6 8}$ \\
$X_{2}$ & 1 & 32.69 & 32.69 & 5.62 & $\mathbf{0 . 0 3 9 2}$ \\
$X_{3}$ & 1 & 747.61 & 747.61 & 128.57 & $<0.0001$ \\
$X_{2} \times X_{1}$ & 1 & 0.88 & 0.88 & 0.15 & 0.7058 \\
$X_{2} \times X_{1}$ & 1 & 5.10 & 5.10 & 0.88 & 0.3709 \\
$X_{2} \times X_{3}$ & 1 & 0.66 & 0.66 & 0.11 & 0.7440 \\
$X_{1}^{2}$ & 1 & 83.59 & 83.59 & 14.37 & $\mathbf{0 . 0 0 3 5}$ \\
$X_{2}^{2}$ & 1 & 7.70 & 7.70 & 1.32 & 0.2767 \\
$X_{3}^{2}$ & 1 & 242.14 & 242.14 & 41.64 & $<0.0001$ \\
Residual & 10 & 58.15 & 5.81 & & \\
Lack of fit & 5 & 48.11 & 9.62 & 4.79 & 0.0552 \\
Pure error & 5 & 10.04 & 2.01 & 20.82 & $<0.0001$ \\
Total & 19 & 1194.04 & 125.95 & 11.08 & 0.0076 \\
\hline
\end{tabular}

Significant values are boldface.

independent variables were effective in the experiments. Other reports have also mentioned the importance of these variables and also confirm the findings of this present study [20]. The polynomial second-order model for response prediction was obtained using multivariate regression analysis. Equation 3 is a polynomial second-order equation, based on the chosen variables, which was developed using the multiple regression analysis of empirical data.

$$
\text { Conversion } \begin{aligned}
(\%)= & 93.39+2.05 X_{1}+1.43 X_{2}+6.84 X_{3} \\
& -1.83 X_{1}^{2}-3.11 X_{3}^{2}
\end{aligned}
$$

Equation 3 suggests that palm oil conversion percentage has a linear second-order relationship with the selected parameters. Positive signs indicate synergic effects and negative signs denote antagonistic effects (i.e. negative effect on conversion percentage). The coefficient of correlation $\left(R^{2}=0.95\right)$ highlights the close relationship between the empirical results and those predicted by the model. These results were similar to those reported in previous studies [20, 32, 33].

According to the results, the effect of MW power on biodiesel production is higher than that of irradiation time and reaction pressure. The effect of reaction pressure on biodiesel production was significant $(p<0.05)$. Increased pressure raised the boiling point of methanol. This helped to keep the methanol in a liquid state during the reaction. Increasing the pressure also slightly increased the number of molecules per volume unit, which in turn gave rise to more collisions. This contributed further to the reaction progress. According to Table 3, the second-order model is statistically significant $(\mathrm{p}<0.0001)$. Pressure-power, 
time-power, and time-pressure interactions were not significant. The RSM diagram of the dependent variable (reaction efficiency) against power and time variations displays contour lines and interactions in a three-dimensional (3D) presentation.

However, as shown in Figure 3B, efficiency initially improved and then declined with increasing pressure in the selected range. This is due to the reversibility of the reaction. It can be observed that the reaction efficiency increases initially (Figure 3A). However, the reaction tends to shift to the left side of the equilibrium, thus reducing the conversion at high MW irradiation time and power. These diagrams show that conversion decreased drastically at longer times, regardless of power level. This may be due to methanol evaporation and its removal from the reaction environment. The study's findings are in complete agreement with the results in the literature [34].

Several authors have reported that MW-assisted biodiesel production is effective due to the combination of exothermicity and the intrinsic properties of dielectric heating. It cannot be said with certainty which has the greatest effect. Nevertheless, the methanol involved in the reaction absorbed MW fairly well. The bipolar properties of methanol caused its molecules to rotate, when exposed to MW exposure, which led to a larger contact surface area between oil and alcohol molecules and thus a faster reaction $[13,15,20,35]$.

Results in Figure 4A indicate that the reagents are exposed to more irradiation at longer reaction times, which accordingly increases the effect of MW on the reaction environment. Transesterification is an equilibrium
A

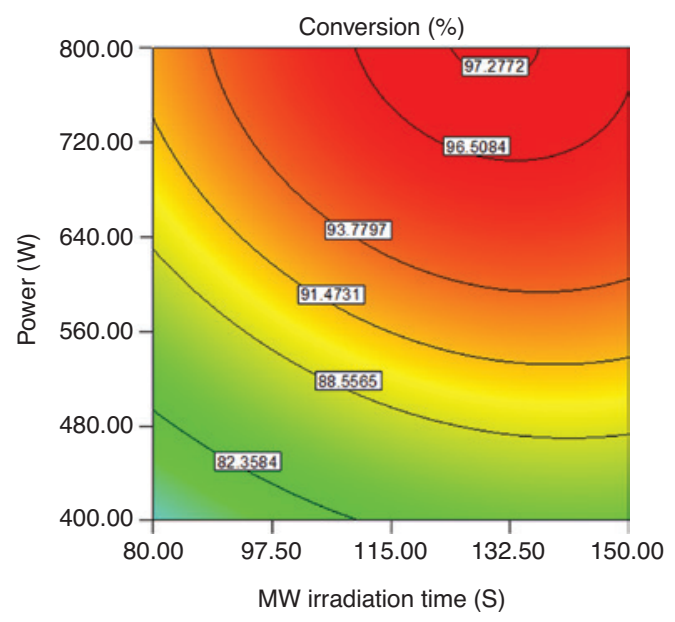

B

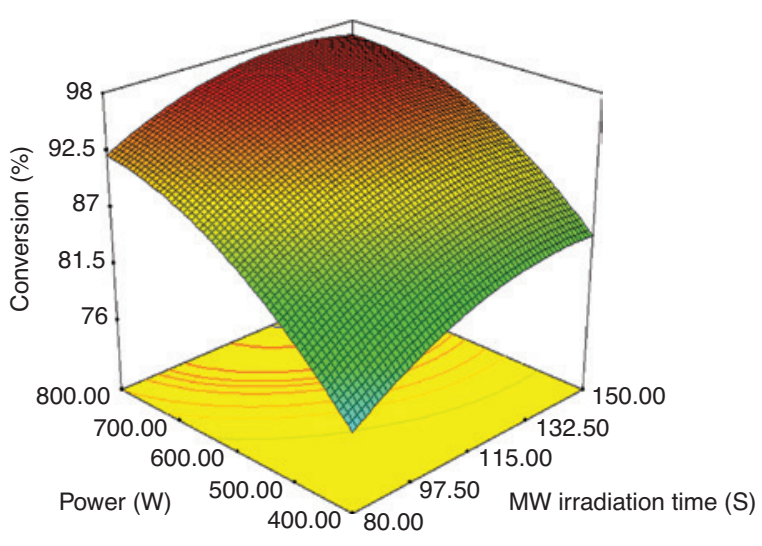

Figure 3: (A) Two-dimensional (2D) and (B) 3D interaction between MW irradiation time and power on FAME conversion.

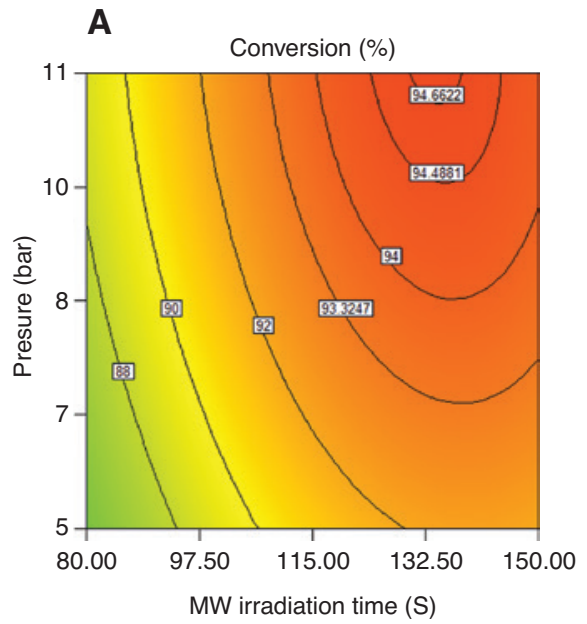

B

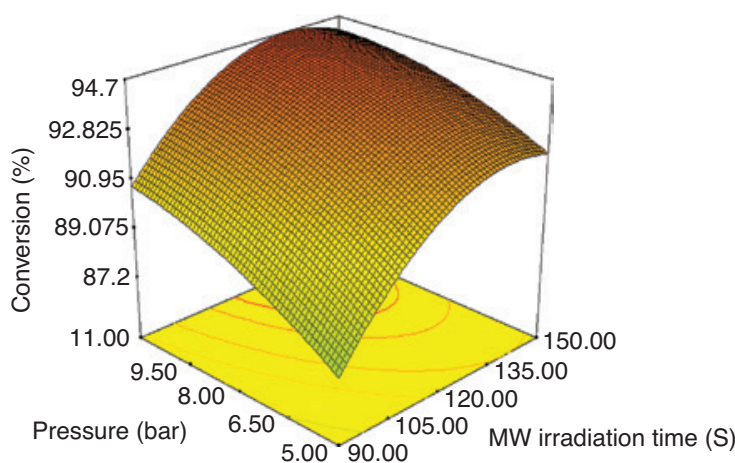

Figure 4: (A) 2D and (B) 3D interaction effect of MW irradiation time and pressure on FAME conversion. 
reaction, meaning that decreased amounts of reagents in the reaction environment lead to reserved reaction and decreases the conversion. Reaction efficiency initially increases and then decreases when irradiation time was extended. Glycerin and methanol are both polar and dissolve in each other, meaning that more methanol is dissolved when more glycerin is produced over a longer time period. This methanol is no longer available for the reaction, causing the reaction to revert to producing methanol, which decreases the total reaction efficiency (Figure 4B). The results indicate that there are a wide range of conditions under which efficiencies higher than $96 \%$, which are required for producing standard biodiesel (Figures 3 and 4), can be reached. As shown in Figure 5A, the model predicted a reaction efficiency of $97 \%$ at $700 \mathrm{~W}$ MW power for 8 bar pressure under the central pressure point.

The optimized process occurred at $138 \mathrm{~s}$ of irradiation at $780 \mathrm{~W}$ under 7 bar pressure. The conversion at this point was $97.82 \%$. An analysis on this optimal point showed a $96.08 \%$ conversion, which is slightly lower than the efficiency obtained (Figure 5B). Previous studies into the consecutive application of MW and ultrasound reported a process time of $30 \mathrm{~min}$. Furthermore, the reported conversion percentage (98.8) and reaction time (30 $\mathrm{min}$ ) in those studies were lower than those reported in this study [36]. Reaction time ( $2 \mathrm{~min}$ ) was also shorter than in other similar studies (5, 4.5 and $6.5 \mathrm{~min})[31,37,38]$.
The physical characteristics of the fatty acid methyl esters (FAME) produced from rapeseed and waste oil are compared and contrasted with those of palm oil in Table 4. All the specifications mentioned are then compared with EN 14214 biodiesel standards. The results show that palm methyl esters meet EN 14214 requirements for biodiesel. Viscosity plays an important role in the typicality of the performance of diesel engine [41]. There are reports on the properties, including viscosity, specific gravity, cetane number, iodine value, and freezing point of the different possible sources for biodiesel, the results of which show that kinematic viscosity ranged between 4 and $5 \mathrm{~mm}^{2} / \mathrm{s}$ [39], whereas the specific gravity of the methyl esters varied between 0.873 and 0.883 . The palm oil in the present work was within the range of parameters that other researchers have obtained $[39,42]$. Comparing the results reported in Table 4 with the other results [43], it can be concluded that biodiesel produced from palm oil has a higher viscosity than others. The kinematic viscosity of palm oil was lower than that of waste oil but higher than rapeseed oil. According to Table 4, rapeseed oil had the highest density, whereas waste oils have the lowest density. The results in the Table 4 also showed that the flash point of palm oil is higher than the others and that the kinematic viscosity of palm oil is lower than that of waste oil and higher than rapeseed oil. In Table 4, rapeseed oil has the highest density, whereas the lowest density belongs to waste oil.
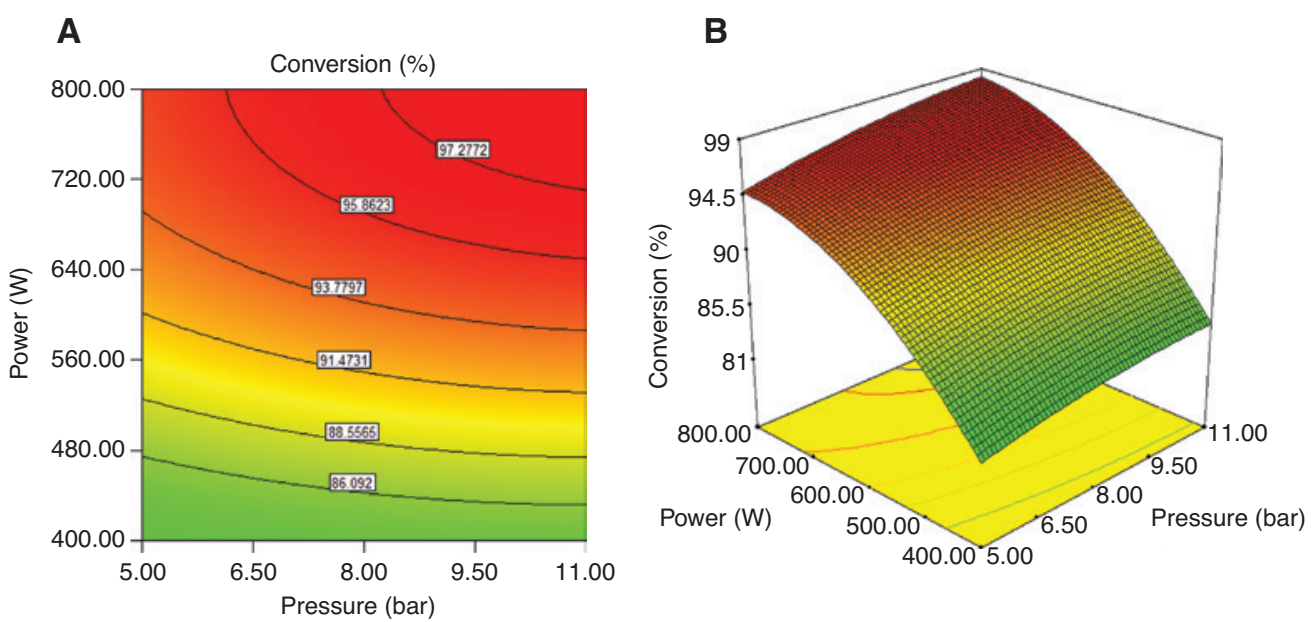

Figure 5: (A) 2D and (B) 3D interaction effect of MW power and pressure on FAME conversion.

Table 4: Comparison of biodiesel produced from palm oil, rapeseed oil, and waste oil.

\begin{tabular}{|c|c|c|c|c|c|}
\hline Specifications & Standard method of test & Waste oil [39] & Rapeseed oil [40] & Palm oil [9] & Unit \\
\hline Flash point & ASTM D92 & 176 & 170 & 179 & ${ }^{\circ} \mathrm{C}$ \\
\hline Kinematic viscosity & ASTM D445 & 4.73 & 4.01 & 4.62 & $\mathrm{~mm}^{2} / \mathrm{s}$ \\
\hline Density & ASTM D1298 & 80 & $900-930$ & 860 & $\mathrm{~kg} / \mathrm{m}^{3}$ \\
\hline
\end{tabular}




\subsection{Validation experiments}

In this study, the conditions of the transesterification reaction under MW irradiation have been studied to optimize biodiesel production efficiency. RSM analyses predicted the optimal point for $96.82 \%$ efficiency as follows: $138 \mathrm{~s}$ MW irradiation, 7 bar pressure, and $780 \mathrm{~W}$ power. Experiments were performed at the RSM-predicted optimal points. Reaction efficiency was $96.71 \%$, which is slightly lower than the model-predicted value. However, this error, which may have been caused by instruments or the operator, was acceptable according to the ANOVA table and significance $(\mathrm{p}<0.05)$.

\section{Conclusions}

RSM has been used to optimize the transesterification reaction conditions for palm oil under MW irradiation. RSM was used to optimize the transesterification reaction conditions for palm oil, which was exposed to pressure and MW irradiation. The MW heating effect had a significant effect on the conversion, which was improved by the ionic rotation phenomenon and dipole rotation. Results suggest that MW power and irradiation time have a significant influence on biodiesel production, at the $1 \%$ level, whereas pressure had significant effects at the 5\% level. Our findings suggest that there are a wide range of experimental conditions under which $96 \%$ efficiency for palm oil transesterification can be achieved. Moreover, increased power and longer irradiation times initially drive the reaction toward producing methyl esters before reaching the optimal point, after which reaction efficiency decreases due to reaction reversibility. The reaction time (138 s; 96.71\% yield) was considerably shorter than in conventional processes (1 h) and sonochemical methods (5 min) [7]. In this work, the total energy consumption (measuring by plug-in meter) for FAME preparation in a pressurized, flow-microwave reactor, was much lower than in conventional processes at $0.88 \mathrm{MJ} / 1$ [DMH pump $(0.64 \mathrm{MJ} / \mathrm{l})$, dielectric heating and mechanical stirrer $(0.24 \mathrm{MJ} / \mathrm{l})$ ] vs. $1.92 \mathrm{MJ} / \mathrm{l}$ ( $400 \mathrm{~W}$ heating and mechanical stirrer for $40 \mathrm{~min}$ ). Biodiesel from all samples fully complied with ASTM D6751 recommendations.

Acknowledgements: This work was supported by the University of Turin (fondi ricerca locale ex-60\% 2015). The authors also wish to thank the Shahid Chamran University of Ahvaz for supporting part of this investigation.

\section{References}

[1] Strzalka R, Schneider D, Eicker U. Renew. Sustain. Energy Rev. 2017, 72, 801-820.

[2] Sharma A, Pareek V, Zhang D. Renew. Sustain. Energy Rev. 2015, 50, 1081-1096.

[3] Najafi G, Ghobadian B, Yusaf TTF. Renew. Sustain. Energy Rev. 2011, 15, 3870-3876.

[4] Dharma S, Hassan MH, Ong HC, Sebayang AH, Silitonga AS, Kusumo F. Chem. Eng. Trans. 2017, 56. doi:10.3303/ CET1756092.

[5] Talebian-Kiakalaieh A, Amin NAS, Mazaheri H. Appl. Energy. 2013, 104, 683-710.

[6] Cintas P, Mantegna S, Gaudino E, Cravotto G. Ultrason. Sonochem. 2010, 17, 985-989.

[7] Choedkiatsakul I, Ngaosuwan K, Cravotto G. Ultrason. Sonochem. 2014, 21, 1585-1591.

[8] Crudo D, Bosco V, Cavagli G, Grillo G, Mantegna S, Cravotto G. Ultrason. Sonochem. 2016, 33, 220-225.

[9] Choedkiatsakul I, Ngaosuwan K, Assabumrungrat S, Mantegna S, Cravotto G. Renew. Energy 2015, 83, 25-29.

[10] Choedkiatsakul I, Ngaosuwan K, Assabumrungrat S, Tabasso S. Biomass Bioenergy 2015, 77, 186-191.

[11] Qiu Z, Zhao L, Weatherley L. Chem. Eng. Process. Process Intensif. 2010, 49, 323-330.

[12] Safieddin Ardebili SM, Hashjin TT, Ghobadian B, Najafi G, Mantegna S, Cravotto G. Green Process. Synth. 2015, 4, 259-267.

[13] Cravotto G, Cintas P. Chemistry-Eur. J. 2007, 13, 1902-1909.

[14] Lin YC, Hsu KH, Lin JF. Fuel 2014, 115, 306-311.

[15] Patil PD, Gude VG, Mannarswamy A, Cooke P, Munson-McGee S, Nirmalakhandan N, Lammers P. Bioresour. Technol. 2011, 102, 1399-1405.

[16] Azcan N, Danisman A. Fuel 2007, 86, 2639-2644.

[17] Patil P, Gude V, Pinappu S, Deng S. Chem. Eng. J. 2011, 168, 1296-1300.

[18] Yuan H, Yang B, Zhu G. Energy Fuels 2008, 23, 548-552.

[19] Yu D, Tian L, Ma D, Wu H, Wang Z, Wang L, Fang X. Green Chem. 2010, 12, 844-850.

[20] Leadbeater N, Stencel L. Energy Fuels 2006, 20, 2281-2283.

[21] Saifuddin N, Chua K. Malaysian J. Chem. 2004, 6, 77-82.

[22] Lertsathapornsuk V, Pairintra R, Aryusuk K, Krisnangkura K. Fuel Process. Technol. 2008, 89, 1330-1336.

[23] Chen K, Lin Y, Hsu K, Wang H. Energy 2012, 38, 151-156.

[24] Li M, Zheng Y, Chen Y, Zhu X. Bioresour. Technol. 2014, 154, 345-348.

[25] Vicente G, Martinez M, Aracil J. Bioresour. Technol. 2007, 98 , $1724-1733$.

[26] Yuan X, Liu J, Zeng G, Shi J, Tong J, Huang G. Renew. Energy 2008, 33, 1678-1684.

[27] Ahmad AL, Ismail S, Bhatia S. Environ. Sci. Technol. 2005, 39, 2828-2834.

[28] Arsenović M, Stanković S, Pezo L, Mančić L, Radojević Z. Ceram. Int. 2013, 39, 3065-3075.

[29] Worapun I, Thaiyasiut P, Pianthong K. SWU Eng. J. 2011, 6, 16-30.

[30] Box G, Draper N. Empirical Model-Building and Response Surfaces, John Wiley \& Sons: Canada, 1987.

[31] Zu Y, Zhang S, Fu Y, Liu W, Liu Z, Luo M. Eur. Food Res. Technol. 2009, 229, 43. 
[32] Zare M, Ghobadian B, Fayyazi E, Najafi G. Int. J. Agric. Crop Sci. 2013, 1314-1317. doi: 10.13140/RG.2.1.1742.0968.

[33] Hincapié GM, Valange S, Barrault J, Moreno JA, López DP. Univ. Sci. 2014, 19, 193-200.

[34] Mazo P, Restrepo G, Rios L. Biodiesel: Quality, Emissions and By-products, InTech: Croatia, 2011, p 21.

[35] Suppalakpanya K, Ratanawilai SB, Tongurai C. Fuel 2010, 89, 2140-2144.

[36] Santos FFP, Rodrigues S, Fernandes FAN. Fuel Process. Technol. 2009, 90, 312-316.

[37] Rathana YS, Roces A, Bacani FT, Tan RR, Kubouchi M, Piyachat Y. Int. J. Chem. React. Eng 2010, 8, 1542-6580.
[38] Kamath V, Regupathi I, Saidutta M. Biofuels 2010, 1, 847-854.

[39] Hoekman S, Broch A, Robbins C, Ceniceros E. Sustain. Energy Rev. 2012, 16, 143-169.

[40] Yahyaee R, Ghobadian B, Najafi G. Renew. Sustain. Energy Rev. 2013, 17, 312-319.

[41] Mofijur M, Rasul MG, Hyde J. Procedia Eng. 2015, 105, 658-664.

[42] Nagi J, Sied KA, Nagi F. Int. Conf. Constr. Build. Technol. (ICCBT 2008). 2008, 79-94.

[43] Barontini F, Simone M, Triana F, Mancini A. Renew. Energy 2015, 83, 954-962. 